\title{
SEMA5A-PLXNB3 axis promotes PDAC liver metastasis outgrowth through enhancing Warburg effect
}

Kun Wang ( $\sim$ kaoyanzyyx@163.com )

The First Affiliated Hospital of Soochow University

Min $\mathrm{He}$

Shanghai Jiao Tong University

Xin Fan

Affiliated Hospital of Jiangsu University

Jian Zhou

The First Affiliated Hospital of Soochow University

Jian Yang

The First Affiliated Hospital of Soochow University

Lin Wang

Affiliated Hospital of Jiangsu University

Zhihong Zhao

Affiliated Hospital of Jiangsu University

Chun Dai

People's Hospital of Yangzhong

Zixiang Zhang

The First Affiliated Hospital of Soochow University

\section{Research Article}

Keywords: Liver metastasis, Pancreatic ductal adenocarcinoma, Warburg effects, Tumor Microenvironment, SEMA5A

Posted Date: January 27th, 2022

DOI: https://doi.org/10.21203/rs.3.rs-1260906/v1

License: (c) (1) This work is licensed under a Creative Commons Attribution 4.0 International License.

Read Full License 


\section{Abstract}

Background: Patients bearing liver metastasis of pancreatic adeno carcinoma (PDAC) suffer from poor prognosis due to its short duration and high mortality. Complex tumor microenvironment (TME) exists in liver metastatic niches and tumor associated macrophages (TAMs) have play vital roles in metastasis generation and outgrowth.

Methods: We set off from investigating the aggregation and infiltration of TAMs at metastasis sites of PDAC and further evaluated related prognosis using clinical samples, discovering the critical roles of SEMA5A-PLXNB3 axis have played in PDAC liver metastasis. We thus performed intrasplenic injection mouse models assessed by $\mathrm{CT}$ combined in vivo imaging with organ reconstruction and Kras $^{\mathrm{G12D}} / \operatorname{Trp} 53^{\mathrm{R} 172 \mathrm{H}} / \mathrm{Pd}$ 1-Cre (KPC) mouse models for in vivo study and CCK-8 assays for in vitro study. In mechanism investigation, we observed the alterations of glucose consumptions, lactate productions and expression of key enzymes involved in glycolysis induced by SEMA5A-PLXNB3 axis. Seahorse system measuring extracellular acid rate (ECAR) was also utilized.

Results: We have demonstrated that M2 TAMs enriched liver metastatic niches predicted poor prognosis. Further studies have unveiled that TAMs derived secreted protein SEMA5A could bind to tumor cell expressed PLXNB3 and thus facilitated the proliferation ability of which. The activation of SEMA5APLXNB3 axis increased glucose consumption and lactate production of tumor cells via modulating location of HIF-1a and stimulating expressions of glycolysis related genes.

Conclusion: We have discovered that SEMA5A-PLXNB3 axis could achieve tumor cell proliferation and survival via enhancing aerobic glycolysis termed as Warburg effects. Targeting this axis may be a potential therapeutic approach for PDAC patients with unresectable liver metastasis.

\section{Introduction}

Pancreatic adeno carcinoma (PDAC) has been one of malignancies with poor prognosis, its high fatality rate and metastasis behavior lead to a 5-year survival rate of less than $6 \%^{1-2}$. The most common target organ for PDAC distant metastasis is liver and patients bearing liver metastasis couldn't get access to surgery and suffer from frequently relapsing. Thus, it is importance to study the mechanism and potential therapeutic targets for liver metastasis of PDAC.

Tumor microenvironment (TME) consists of complex members including cancer associated fibroblasts $(\mathrm{CAFs})^{3-4}$, tumor associated macrophages (TAMs) ${ }^{5-7}$ and regulatory T cells (T-regs) $)^{8-10}$, etc., which share reciprocal functions to each other. Lots of study have been done to emphasize the importance of TME, while the relationship of TAMs and liver metastatic niches of PDAC remains largely unknown. TAMs usually aggregated around tumor niches into enriched extracellular matrix (ECM) or even deeply infiltrated into the intervals of niches to exert their promotive or anti effects on tumor cells ${ }^{11-12}$. Accumulated evidences have demonstrated that M2 type macrophages could stimulate proliferation, 
migration, and invasion activity of tumor cells mainly via secreting various chemokines or other proteins. $^{5-6,11,13}$ Tumor-growth promotive protein from M2 TAMs could be detected at high concentration to immerse tumor cells for niche filtration and expansion.

Axon guidance family is vital in embryonic development and neuron growth, which can act as a leader to decide and induce the directions of axons of neurons ${ }^{14}$. While in recent years, more and more reports have discovered that this family has played critical roles in tumorigenesis and metastasis ${ }^{15-16}$. Among them, semaphorin is one of the most important family mentioned in tumor related pathways ${ }^{16-17}$. Semaphorin is mainly recognized responsible for extracellular signaling transduction and also essential for cell development and even tumor growth ${ }^{18-19}$. Diverse structures of SEMA family members are detected so far and their cellular locations differ from each other. It has been discovered that SEMA2 and 3 family members were all secreted proteins; while though SEMA5 family members were usually considered as transmembrane proteins, several studies have demonstrated that they would be cleaved and released into cellular matrix ${ }^{20}$. The canonical receptors of SEMAs were PLXNs, which were expressed on the membrane of cells and received the signals from SEMAs before triggering the downstream pathway.

In this study, we set off from evaluating the infiltration of M2 TAMs in patients derived liver metastasis tissues and discovered the enrichment of extracellular SEMA5A in M2 TAMs aggregation area. We also found that PLXNB3, one of the canonical receptors of SEMA5A, was also aberrantly overexpressed on M2 TAMs infiltrated metastatic niche resident tumor cell membranes. We established intrasplenic injection mouse model to mimic the physiological progress of PDAC liver metastasis and assessed by CT combined in vivo imaging with organ reconstruction. We also generated $K r a s^{G 12 D} / \operatorname{Trp} 53^{\mathrm{R} 172 \mathrm{H}} / \mathrm{Pd} \times 1$-Cre (KPC) mouse models which could form liver metastasis of PDAC spontaneously. In mechanism exploration, we discovered that SEMA5A-PLXNB3 axis efficiently facilitated Warburg effects via upregulating cell stress related genes and glycolysis function involved key enzymes, thus promote tumor cells growth.

Our study has put forward a novel insight into liver metastasis of PDAC and might be a promising target for metastatic PDAC.

\section{Material And Methods}

\section{Constructs \& Reagents.}

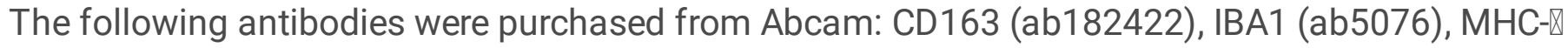
(ab55152), CD11c (ab52632), SEMA5A (ab127002) \& (ab51957), CK19 (ab52625), PLXNB3 (ab204172), PLXNA3 (ab41564), PLXNA3 (ab39350), Ki67 (ab15580).

The following antibodies were purchased from Jackson: anti-rabbit Alexa Fluor 488 (111-545-003), 596 (111-585-003) and anti-mouse Alexa Fluor 488 (111-545-003), 596 (111-585-003); HRP-conjugated 
secondary antibodies (115-035-003 and 111-035-003)

The following reagent was purchased from Dojindo Molecular Technologies: Cell Counting Kit-8 (CCK-8).

The following reagent was purchased from Life Technologies: G-Dynabeads (10004D).

The following reagents were purchased from Roche: In Situ Cell Death Fluorescein Kit Tunel (11684795910) and SYBR Premix Ex Taq $(04,913,914,001)$

The following reagents were purchased from Sigma-Aldrich: Glucose assay kit, oligomycin and 2deoxyglucose (2-DG, D8375).

The following reagents were purchased from BioVision: Lactate Assay Kit

The following reagents were purchased from Cell Signaling Technologies: DAB (8059)

\section{Clinical samples}

Clinical samples involved in this study were all from Renji hospital, Shanghai Jiaotong University School of Medicine. We claimed that all samples used in our study (the sample collections, tissue preparations and experiments performance, etc.) were approved by local ethics committee, the approval number is RA2019-116.

All these patients were diagnosed by both clinical surgeons and professional pathologists.

Our study on liver metastasis containing 20 cases patients with liver metastasis of PDAC. The sample of liver metastasis were collected for IHC-P staining, IF staining or real- time PCR.

\section{Animals}

Male C57BL/B mice (6-8 weeks) and KPC mice (about 3-4 months) used in this study were all received all received humane care, housed, and fed in accordance with the Guide for the Care and Use of Laboratory Animals prepared by the National Academy of Sciences and published by the National Institutes of Health. Our animal experiments were approved and invested under approved protocol number 20141204 assigned by the Research Ethics Committee of East China Normal University.

\section{Immunohistochemistry and Immunofluorescence}

Human liver metastasis samples or mouse model liver metastasis samples were collected and performed paraformaldehyde fixation. At the beginning, xylene in gradient alcohol at different concentration (from $100 \%$ to $75 \%$ ) were used to perform deparaffinization and rehydration. Citrate buffer at $100^{\circ} \mathrm{C}$ was used for antigen retrieval which lasted $20 \mathrm{~min}$. Then endogenous peroxidases inactivation using exposure of 
$0.3 \%$ hydrogen peroxide in methanol to slides was performed. After PBS washing, bovine serum albumin (BSA) was used to blocking for $1.5 \mathrm{~h}$ before incubation of primary antibodies overnight. For IHC-P staining, HRP conjugated secondary antibodies exposure was performed $2 \mathrm{~h}$ at room temperature. After washing 3 times, DAB staining was performed about 10s-30s according to target protein enrichment. Hematoxylin staining was then performed on all slides followed by 10 min washing. For IF staining, the IF secondary antibodies were performed and nuclei was stained with DAPI.

\section{Cell culture}

In this study, human PDAC cell lines PANC1, BxPC3, AsPC1 and CAPAN1, human normal pancreatic cells HPNE and murine PDAC cell lines Panc02 and KPC1199 were all purchased from Cell Bank of Chinese Academy of Sciences (China). All these cell lines were culture and maintained according to official instructions. The complete medium consists of DMEM medium, $10 \%$ fetal bovine serum (FBS) and $1 \%$ antibiotic mixture. All cells were kept at $37^{\circ} \mathrm{C}$ with $5 \% \mathrm{CO}_{2}$.

\section{SEMA5A and SEMA5A- $\triangle T$ TSP protein expression and purification}

We utilized episomal expression vector with pCEP-Pu-Strep II-tag carrying SEMA5A or SEMA5A- $\triangle \mathrm{TSP}$. 293-T cells transfected with these reconstructed plasmids were then filtered by puromycin at $5 \mu \mathrm{g} / \mathrm{ml}$. Then the cell supernatants were collected for application to Strep Tactin sepharose column. Then, columns were washed by binding buffer. At last, elution buffer containing $2.5 \mathrm{mM}$ desthiobiotin was used for harvesting SEMA5A or SEMA5A- $\triangle T$ SP. The concentrations of these two proteins were determined by Nanodrop 2000.

\section{Cell transduction}

The lentivirus carrying shRNA targeting PLXNB3 was performed (5'-AGCAGATGGTGGAGAGGT A-3'). 1× HitranasG transfection reagent was utilized for transfection. Then the transfected tumor cells were filtered by puromycin at a concentration of $5 \mu \mathrm{g} / \mathrm{ml}$ fist and maintained at $2 \mu \mathrm{g} / \mathrm{ml}$.

\section{Cell viability assay}

Cell counting kit-8 was performed to evaluate the tumor cell viability. Robust PANC1 at 5000 cells/100 $\mathrm{\mu l}$,

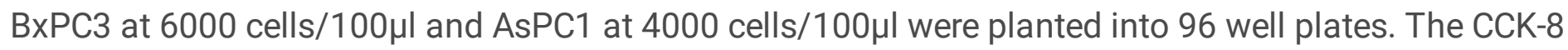
reagent was diluted by complete medium at a ratio of $1: 10$ for $1 \mathrm{~h}$. Then microplate reader was performed to measure the incubated holes at $450 \mathrm{~nm}$. 


\section{Real-time PCR}

RNA extraction was performed before cDNAs were transcribed using PrimeScript RT-PCR kit. SYBR green ex taq was used for 7500 Real-time PCR system (Applied Biosystems, USA). Sequences of primers are as follow: 


\begin{tabular}{|c|c|c|}
\hline Gene & Forward primer ( $5^{\prime}$ to $\left.3^{\prime}\right)$ & Reverse primer (5' to $\left.3^{\prime}\right)$ \\
\hline SMEA5A & GAACCGGAAGCGTGTT & CAGTGAGATGTGGGTTGAAG \\
\hline PLXNB3 & GTGCGGAACCTTCAACATTT & AAAGAGCATGGGTGTTGTCC \\
\hline SEMA3A & GTGCCAAGGCTGAAATTATCCT & СCСАCTTGCATTCATСТСТTСТ \\
\hline SEMA3B & ACATTGGTACTGAGTGCATGAAC & GCCATCCTCTATCСTTCCTGG \\
\hline SEMA3C & TTTGCGTGTTGGTTGGAGTAT & TCCTGTAGTCTAAAGGATGGTGG \\
\hline SEMA3D & GCAAAGGAACGGGTGGAATTA & TCTGCCAGACTCCAAATTATGTG \\
\hline SEMA3E & AGGCAGGGACCTTGTATATTCC & TGTACTCGGCCAGTGTATCTC \\
\hline SEMA3F & AACACAACCGACTACCGAATC & GGCTGCCCAGTGTATAATGAG \\
\hline SEMA3G & CTCTGCGTTCCGACTCTGAC & CTGACAGTGACATGGTTCGAG \\
\hline SEMA5B & CCGTGGGTCTCTAACTTCACC & GACTCGCACGTAGTTCTGACA \\
\hline PLXNA1 & ACCCACCTAGTGGTGCATGA & CGGTTAGCGGCATAGTCCA \\
\hline PLXNA2 & CTGAGAATCGTGACTGGACCT & GCTTATAGACCCGGTTGATGG \\
\hline PLXNA3 & AGTCCTGCTATCGTGGGGAG & CAGAAGTTGCCGTTGATCTGC \\
\hline PLXNA4 & GTCATTTGTCACATTCCGAGGA & GCTTGTAAATCCGATTGACGGC \\
\hline PLXNB1 & ACCAАCTGCATTCACTCCCAА & GCACTCATCAGGCATCACAG \\
\hline PLXNB2 & AGCCTCTTCAAGGGCATCTG & GCCACGAAAGACTTCTCCCC \\
\hline GLUT1 & ATTGGCTCCGGTATCGTCAAC & GCTCAGATAGGACATCCAGGGTA \\
\hline HK2 & TGATCGCCTGCTTATTCACGG & AACCGCCTAGAAATCTCCAGA \\
\hline LDHA & GCTCCCCAGAACAAGATTACAG & TCGCCCTTGAGTTTGTCTTC \\
\hline PKM & TCGCATGCAGCACCTGATT & CCTCGAATAGCTGCAAGTGGTA \\
\hline GPI1 & CAAGGACCGCTTCAACCACTT & CCAGGATGGGTGTGTTTGACC \\
\hline PFKL & GGTGCCAAAGTCTTCCTCAT & GATGATGTTGGAGACGCTCA \\
\hline ALDOA & ААСТTТССТСТGССТАGССС & GTACAGGCACAGTCGCAGAG \\
\hline TPI1 & AGCTCATCGGCACTCTGAAC & CCACAGCAATCTTGGGATCT \\
\hline GAPDH & CTGGGCTACACTGAGCACC & AAGTGGTCGTTGAGGGCAATG \\
\hline PGK2 & AAACTGGATGTTAGAGGGAAGCG & GGCCGACCTAGATGACTCATAAG \\
\hline PGAM2 & AGAAGCACCCCTACTACAACTC & TCTGGGGAACAATCTCCTCGT \\
\hline EN01 & GCCGTGAACGAGAAGTCCTG & ACGCCTGAAGAGACTCGGT \\
\hline
\end{tabular}




\section{Seahorse assay}

The ECAR measurement was performed on Seahorse XF96 Flux Analyser (Seahorse Bioscience). PANC1 at 15000 cells and BxPC3 at 20000 cells were seeded on XF-96-wll plate. Then these cells were deprived from FBS for starvation for 24h. In the following-up, these cells were exposed in subsequence to glucose $(10 \mathrm{mM})$, oligomycin $(1 \mathrm{mM})$ and 2-deoxyglucose $(80 \mathrm{mM})$. The related data would be recorded.

\section{Intrasplenic mouse model and CT combined in vivo imaging with organ reconstruction}

For injection preparation, murine PDAC cell line derived from KPC mice, Kpc1199 was performed.

Kpc1199 experienced stable transfection of luciferase was cultured before prepared at a concentration of $2 \mathrm{e} 6$ cells in $20 \mu \mathrm{l}$ medium without FBS. Then mice under $2.5 \%$ isoflurane inhalation anesthesia were exerted surgery for exposure of spleen followed by cell injection via an insulin syringe. For in vivo imaging, mice bearing liver metastasis were intraperitonially injected $50 \mathrm{mg}$ D-luciferin $(200 \mu \mathrm{L})$ before anesthetized with 2.5\% vaporized inhaled isoflurane. In Vivo Imaging System (IVIS) Spectrum (Caliper Life Sciences, Waltham, MA) was used for detecting metastatic niches. The CT image and organ reconstruction were generated and merged by Ai of this system.

\section{Ex-vivo assay}

For ex-vivo assay, fresh metastasized liver of KPC mice were harvested before experiment. The liver containing metastatic niches were sliced in half on ice to ensure that the histological characteristics of left part $(L)$ and right part $(R)$ were same. Then

$L$ part was placed in preheated complete medium with administration of rSEMA5A (30nM), while R part was place in medium with administration of rSEMA5A $\triangle T S P(30 n M)$. The duration is 2 hours. Then tissues were collected for further analysis.

\section{Glucose uptake and lactate production measurement}

All cells involved in this test were planted in 6-well plate and performed 24h starvation before tests. Then glucose assay kit and lactate assay kit were performed. All experiments were performed according to manufacturer's instructions.

\section{Data analysis}


All data performed in this study were treated by SPSS 14.0 and GraphPad 7.0

\section{Results}

\section{SEMA5A is derived from infiltrated M2 TAMs}

To uncover the how TAMs affect tumor cell metabolism thus leads to poor prognosis of hepatically metastatic PDAC, we first set out to investigate the category and distribution of TAMs. IHC-P on liver metastatic niches of patients bearing PDAC have demonstrated that IBA1 or CD163 positive M2 TAMs have predominated in TAMs infiltrating niches compared to MHCII or CD11c expressed ones (Figure 1A). Especially, the SUV of PET-CT in M2 TAMs severely infiltrated were much higher than those with little infiltration (Figure 1B), which indicated relationship between cell metabolism and M2 TAMs distributions. Moreover, the aggregation and infiltration of M2 TAMs could also lead to poor prognosis of metastasized PDAC (Figure 1C). It has been reported that secreted SEMA family has played vital roles in metastasis of several kinds of cancers and closely related to TME, we next studied the expressions of which in liver metastasis with or without M2 TAMs infiltration. Interestingly, some SEMA family members including SEMA3A and SEMA3E have displayed equal high expression in both groups, indicating that the source of which was not TAMs; while SEMA5A, another important secreted ligand, has been found to upregulate in TAMs enriched metastasis tissues (Figure 1D). Further confirmation on liver metastasis slices were also performed, the results of which were in accordance with our formed discovery (Figure 1E). We also compared the relationship of CD163 and SEMA5B, another SEMA5 family member, in liver metastasis samples and gained that only SEMA5A possessed strong relationship with M2 TAMs (Figure 1F and 1G).

Finally, we also performed IF staining to find that SEMA5A was derived from CD163 positive M2 TAMs (Figure $1 \mathrm{H}$ ).

Taken together, we have discovered that M2 TAMs would lead to poor prognosis in PDAC patients and act as a source of SEMA5A.

\section{SEMA5A is collocated with its receptor PLXNB3 receptor in tumor}

We next aimed to find out the receptor of SEMA5A in PDAC liver metastasis. It has been concluded that the potential receptors were including PLXNA3, PLXNA4 and PLXNB3, which were discovered upregulated in SEMA5A highly expressed niches in varying degrees (Figure 2A). We next performed IF staining on these tissues and demonstrated that it was PLXNB3 which located on CK19+ metastasized tumor cells in niches (Figure 2B). What's more, we also illustrated that SEMA5A was enriched in stoma around PLXNB3 expressed niches (Figure 2C). We have also calculated the relationship of SEMA5A and its three potential receptors in PDAC liver metastasis, the results have demonstrated our hypothesis (Figure 2D). Furthermore, we have assessed their relationships via scoring their IHC-P staining and got that PLXNB3 was co-expressed with either CD163 or SEMA5A in PDAC liver metastasis tissues (Figure 2E-2F). At last, 
we have selected 16 PDAC patients whose liver metastasis niches were of high SEMA5A abundance and divided them into two groups according to PLXNB3 expressions before prognosis assessment. Results have elucidated that the dual high expression of SEMA5A and PLXNB3 would lead to poor prognosis (Figure 2G).

In summary, these results have shown that SEMA5A and its receptor PLXNB3 were discovered enhanced co-expression in liver metastasis of PDAC and predicted poor prognosis.

\section{SEMA5A-PLXNB3 axis promoted liver metastasis in vivo}

We first performed IF staining on KPC mice in which liver metastasis would generated spontaneously at about 4 months old. The metastatic niches of KPC mice performed double positive of CK19 and PLXNB3, indicating that TME of liver metastatic niches of KPC mice was similar to that of patients (Figure $3 \mathrm{~A}$ ). Then we examined SEMA5A and PLXNB3 expressions in PDAC human or murine cell lines (Figure 3B). It has been noticed that PLXNB3 were upregulated in most cell lines, indicating the tumor resident behavior of which; while SEMA5A was maintained at a relative low level for its source was usually exogenous. Then intrasplenic injection model was performed. In this model, the injected tumor cells would begin to generated metastatic niches from left lobe in liver after passing through the portal vein in accordance with physiological process of PDAC liver metastasis (Figure 3C). Metastasis burdens of C57BL/6 mice bearing KPC1199 injection were evaluated via CT combined in vivo imaging with organ reconstruction and results of which have demonstrated that RNAi of $P L X N B 3$ attenuated the outgrowth of liver metastasis (Figure 3C-3F). Interestingly, the knockdown of PLXNB3 also reduced the infiltration of CD163 positive M2 TAMs into metastatic niches, illustrating that the promotion effects of tumor cells and TAMs were reciprocal (Figure 3G). Further staining also elucidated that PLXNB3 could facilitate cell proliferation and prevent tumor cells from apoptosis (Figure $3 \mathrm{H}$ ). Meanwhile, $\mathrm{KM}$ analysis on mice bearing liver metastasis showed that PLXNB3 knockdown significantly prolong life survival (Figure 3I).

Collectively, these data have demonstrated that PLXNB3 facilitated tumor burden growth in vivo.

\section{SEMA5A-PLXNB3 axis promoted liver metastasis in vitro}

Data have pointed out that PANC1 and BxPC3 cell line highly expressed PLXNB3 with SEMA5A deficiency, we next aim to stimulate these two cell lines with conditional medium (CM) of M1 or M2 TAMs. Human THP-1 cell were cultured before phorbol 12-myristate 13-acetate (PMA) treatment followed by polarization via LPS or IFN-y inducement. CCK-8 assays have shown that M1 CM failed to facilitate tumor cell growth as M2 CM did, while the inference of $P L X N B 3$ could abolish the promotion effect brought by M2 CM (Figure 4A-4D). Furthermore, the M2 TAMs CM would lose its growth stimulation effects on PANC1 tumor cell once SEMA5A was eliminated via immune precipitation (IP) (Figure 4E), which have manifested the important roles of SEMA5A in PLXNB3 induced proliferation advantage. 
SEMA5A protein contains several domains and TSP repeats are responsible for the interaction of SEMA5A to PLXNB3 receptor, cutting off which could greatly abate the binding ability of SEMA5A (Figure $4 F$ ). We next produced recombined SEMA5A (rSEMA5A) and trunked SMEA5A lacking TSP domain (rSEMA5A- $\triangle \mathrm{TSP}$ ) for further study ${ }^{21-22}$. Results of CCK-8 and EdU assay have indicated that administration of rSEMA5A could significantly enhance the proliferation ability of PLXNB3 overexpressed tumor cell AsPC1 ${ }^{P L X N B 3-O E}$, while this effect would be lost once TSP domain was cutting off (Figure 4G$4 \mathrm{H})$. What's more, the removement of FBS could induce the apoptosis of tumor cells with vehicle or SEMA5A- $\triangle \mathrm{TSP}$ exposure, while the apoptosis effects were significantly attenuated with treatment of rSEMA5A (Figure 4I-4J).

Taken together, these results have indicated that the binding of SEMA5A to PLXNB3 is critical for its proliferation promotive effects.

\section{SEMA5A-PLXNB3 axis facilitates tumor cell growth via enhancing Warburg effect}

We next aimed to unveil the mechanism of SEMA5A-PLXNB3 axis mediated cell proliferation effects. We have observed that activated SEMA5A and PLXNB3 axis could enhance the survival ability in medium lacking FBS (Figure 4I-4J), and further study have shown that this axis activation could induce the expression of HIF1A and C-MYC which is vital for cell against stress including hypoxia (Figure 5A-5B). To validate these results, we performed ex vivo tests. PDAC tumor cells metastasized livers of KPC mice were sliced in half, one was immersed into complete medium with rSEMA5A and another one was treated with rSEMA5A- $\triangle T S P$. IF staining has displayed that the exposure of rSEMA5A significantly increased the expression of HIF1A and promoted its nuclear location (Figure $5 \mathrm{C}$ ). Warburg effects is charactered by series abnormal cell activities, including enhanced glucose consumption, additional lactate production and aberrant extracellular $\mathrm{H}^{+}$accumulation, etc. We have demonstrated that treatment of rSEMA5A on PLXNB3 expressed tumor cell lines upregulated glucose consumption and lactate production (Figure 5D$5 \mathrm{E})$. We have also explored the expression alterations of key enzymes involved in glycolysis, the activation of this axis could upregulate the expressions of most enzymes in glycolysis and hypoxia pathway (Figure 5F-5G). At last, we consolidated these results in Seahorse system, the results have shown that activated SEMA5A-PLXNB3 axis significantly facilitate the extracellular acid rate (ECAR) value (Figure $5 \mathrm{H}-5 \mathrm{l})$.

Altogether, our results have push forward evidence that SEMA5A-PLXNB3 axis promote tumor cell proliferation via enhancing Warburg effect.

\section{Discussion}

In our study, we have demonstrated that M2 TAMs derived secreted protein SEMA5A would interact with tumor cell membrane located PLXNB3 and thus induced tumor cell proliferation for metastatic niche 
expansion via enhancing Warburg effects. SEMA family has long been recognized as vital in various kinds of cancers including PDAC, breast cancer and gastric cancer (GC), etc. Here, we mainly research the roles SEMA5A played in liver metastasis of PDAC.

TAMs are essential components of TME, our results on IHC-P have displayed that numerous $\mathrm{CD} 163^{+} \mathrm{M} 2$ TAMs could be observed staying in ECM of liver metastatic niches. The effects they exert on tumor cells was through secreting SEMA5A. The sources of SEMA family were diverse: CAFs derivation, tumor autocrine and immune cell secretion. Through IHC-P staining, PCR screening and IF colocation, we then determined that in liver metastasis of PDAC, the source of SEMA5A was M2 TAMs. The relationship analysis also supported our hypothesis. Considering that multiple receptors of SEMA5A could exist in metastatic niches, we also detected potential receptors PLXNA3 and PLXNA4 of SEMA5A ${ }^{17-18,20}$. IF tests have demonstrated that only PLXNB3 existed on M2 TAMs infiltrated niches and this receptor could be observed co-location in tumor cells and adjacent ECM. We finally confirmed that it was SEMA5A-PLXNB3 axis activated in M2 TAMs dominated niches.

We performed confirmation on both intrasplenic injection mouse model and spontaneous KPC liver metastasis model to evaluate the function of SEMA5A-PLXNB3 axis. Notably, in liver metastatic niches of KPC mice, the stroma is thicker than that in artificial mouse model, thus the upregulation of PLXNB3 in metastatic niches is more persuasive. The outgrowth facilitation PLXNB3 exerted on tumor cells was triggered by SEMA5A was also consolidated by SEMA5A- $\triangle T$ SP. SEMA5A- $T$ TSP which lacked the TSP domain and loses most affinity with PLXNB3, would fail to efficiently bind to PLXNB3 and induce the downstream cell functions, which further provide solid evidence for SEMA5A-PLXNB3 axis.

In mechanism exploration, we have found that this axis could facilitate either the proliferation ability or survival of tumor cells, especially in cell stress. It has been reported that the tumor cells always suffer from fierce struggles for survival due to deficient nutrition, high cell density and relative low $\mathrm{pH}$ value in $\mathrm{TME}^{23-26}$. The situation will be even worse in metastatic TME, since alien microenvironments for tumors are harsh for their implantation and outgrowth. Tumor cell will utilize glucose mainly by aerobic glycolysis rather than oxidative phosphorylation even the oxygen supplement is sufficient, though the latter is much more energetically efficient on generating adenosine triphosphate (ATP). This phenomenon is term as Warburg effect ${ }^{26-29}$. Tumor cells choose Warburg effect not only to meet their needs of rapid energy supplement for exuberant proliferation and growth, but also for gaining enough glycolytic intermediates into various biosynthetic pathways to assemble newly birthed cells ${ }^{30-31}$. Another hypothesis has also unveiled that large amounts of $\mathrm{H}^{+}$were generated as by products and released into TME, causing microenvironment remodeling which could be more adaptive for tumor cells. Thus, enhanced glucose uptake, high concentration of extracellular lactate and aberrantly upregulated glycolysis enzyme are obvious hallmarks of Warburg effect. In our study, we have manifested that activation of SEMA5A-PLXNB3 axis significantly increased the consumption of glucose, lactate production of PDAC cell lines and stimulated the expression of key enzymes involved in glycolysis 
pathway. The binding of SEMA5A to PLXNB3 also upregulated ECAR value, further confirmed our hypothesis.

Our study provided evidence that SEMA5A-PLXNB3 axis activation promotes liver metastasis of PDAC via enhancing Warburg effects, which could be a potential new target for metastasized PDAC therapy.

\section{Conclusion}

In conclusion, we have demonstrated that TAMs aberrantly infiltrated at liver metastasis sites were of M2 type and led to poor prognosis. TAMs derived secreted protein SEMA5A could interact with PLXNB3 positive tumor cells and thus triggered outgrowth of metastatic niches. We have illustrated that activation of SEMA5A-PLXNB3 axis could efficiently trigger outgrowth of tumor cells both in vivo and in vitro. We also elucidated that SEMA5A-PLXNB3 axis could enhance Warburg effect of tumor cells for promotion of their survival and proliferation ability and the mechanism of which is the upregulation of glycolysis key enzymes, leading to increased glucose consumption and lactate production. Our study might provide a new therapeutic strategy for PDAC patients bearing liver metastasis via targeting SEMA5A-PLXNB3 axis.

\section{Declarations}

\section{Ethics approval and consent to participate}

We claimed that all samples used in our study (the sample collections, tissue preparations and experiments performance, etc.) were approved by local ethics committee, the approval number is RA2019-116.

\section{Consent to publication}

$\mathrm{N} / \mathrm{A}$

\section{Availability of data and material}

N/A

\section{Competing interests}

The authors declare no conflict of interest.

\section{Funding}

This study was supported by the National Natural Science Foundation of China (81902385). 


\section{Author contributions}

K.W. and Z.X.Z. were responsible for concept and experimental design; M.H. were responsible for clinical samples collection; J.Z and K.W. were responsible for animal experiments; J.Y and L.W. performed cell molecular biology experiments, Z.H.Z., C.D. and K.W. were responsible for data measurement and scientific writing.

\section{Acknowledgments}

We thank Dr. Xiaoxin Zhang for her kind assistances.

\section{References}

1. Wolfgang, C. L.; Herman, J. M.; Laheru, D. A.; Klein, A. P.; Erdek, M. A.; Fishman, E. K.; Hruban, R. H., Recent progress in pancreatic cancer. CA Cancer J Clin2013, 63 (5), 318-48.

2. Fesinmeyer, M. D.; Austin, M. A.; Li, C. I.; De Roos, A. J.; Bowen, D. J., Differences in survival by histologic type of pancreatic cancer. Cancer Epidemiol Biomarkers Prev2005, 14 (7), 1766-73.

3. Li, Q.; Zhu, C. C.; Ni, B.; Zhang, Z. Z.; Jiang, S. H.; Hu, L. P.; Wang, X.; Zhang, X. X.; Huang, P. Q.; Yang, Q.; Li, J.; Gu, J. R.; Xu, J.; Luo, K. Q.; Zhao, G.; Zhang, Z. G., Lysyl oxidase promotes liver metastasis of gastric cancer via facilitating the reciprocal interactions between tumor cells and cancer associated fibroblasts. EBioMedicine2019, 49, 157-171.

4. Erez, N., Fibroblasts form a hospitable metastatic niche in the liver. Nat Cell Bio/2016, 18 (5), 465-6.

5. Qian, B. Z.; Pollard, J. W., Macrophage diversity enhances tumor progression and metastasis. Cel/2010, 141 (1), 39-51.

6. Mi, X.; Xu, R.; Hong, S.; Xu, T.; Zhang, W.; Liu, M., M2 Macrophage-Derived Exosomal IncRNA AFAP1AS1 and MicroRNA-26a Affect Cell Migration and Metastasis in Esophageal Cancer. Mol Ther Nucleic Acids2020, 22, 779-790.

7. Zhang, Y. L.; Li, Q.; Yang, X. M.; Fang, F.; Li, J.; Wang, Y. H.; Yang, Q.; Zhu, L.; Nie, H. Z.; Zhang, X. L.;

Feng, M. X.; Jiang, S. H.; Tian, G. A.; Hu, L. P.; Lee, H. Y.; Lee, S. J.; Xia, Q.; Zhang, Z. G., SPON2 Promotes M1-like Macrophage Recruitment and Inhibits Hepatocellular Carcinoma Metastasis by Distinct Integrin-Rho GTPase-Hippo Pathways. Cancer Res2018, 78 (9), 2305-2317.

8. Tanaka, A.; Sakaguchi, S., Regulatory T cells in cancer immunotherapy. Cell Res2017, 27 (1), 109118.

9. Petrausch, U.; Poehlein, C. H.; Jensen, S. M.; Twitty, C.; Thompson, J. A.; Assmann, I.; Puri, S.; LaCelle, M. G.; Moudgil, T.; Maston, L.; Friedman, K.; Church, S.; Cardenas, E.; Haley, D. P.; Walker, E. B.; Akporiaye, E.; Weinberg, A. D.; Rosenheim, S.; Crocenzi, T. S.; Hu, H. M.; Curti, B. D.; Urba, W. J.; Fox, B. A., Cancer immunotherapy: the role regulatory $T$ cells play and what can be done to overcome their inhibitory effects. Curr Mol Med2009, 9 (6), 673-82. 
10. Zou, W., Regulatory T cells, tumour immunity and immunotherapy. Nat Rev Immuno/2006, 6 (4), 295307.

11. Sangaletti, S.; Di Carlo, E.; Gariboldi, S.; Miotti, S.; Cappetti, B.; Parenza, M.; Rumio, C.; Brekken, R. A.; Chiodoni, C.; Colombo, M. P., Macrophage-derived SPARC bridges tumor cell-extracellular matrix interactions toward metastasis. Cancer Res2008, 68 (21), 9050-9.

12. Gao, Y.; Xiao, Q.; Ma, H.; Li, L.; Liu, J.; Feng, Y.; Fang, Z.; Wu, J.; Han, X.; Zhang, J.; Sun, Y.; Wu, G.; Padera, R.; Chen, H.; Wong, K. K.; Ge, G.; Ji, H., LKB1 inhibits lung cancer progression through lysyl oxidase and extracellular matrix remodeling. Proc Natl Acad Sci U S A2010, 107 (44), 18892-7.

13. Nielsen, S. R.; Quaranta, V.; Linford, A.; Emeagi, P.; Rainer, C.; Santos, A.; Ireland, L.; Sakai, T.; Sakai, K.; Kim, Y.-S.; Engle, D.; Campbell, F.; Palmer, D.; Ko, J. H.; Tuveson, D. A.; Hirsch, E.; Mielgo, A.; Schmid, M. C., Macrophage-secreted granulin supports pancreatic cancer metastasis by inducing liver fibrosis. Nature cell biology2016, 18 (5), 549-560.

14. Suter, T.; Jaworski, A., Cell migration and axon guidance at the border between central and peripheral nervous system. Science2019, 365 (6456).

15. Jurcak, N. R.; Rucki, A. A.; Muth, S.; Thompson, E.; Sharma, R.; Ding, D.; Zhu, Q.; Eshleman, J. R.; Anders, R. A.; Jaffee, E. M.; Fujiwara, K.; Zheng, L., Axon Guidance Molecules Promote Perineural Invasion and Metastasis of Orthotopic Pancreatic Tumors in Mice. Gastroenterology2019, 157(3), 838-850 e6.

16. Biankin, A. V.; Waddell, N.; Kassahn, K. S.; Gingras, M. C.; Muthuswamy, L. B.; Johns, A. L.; Miller, D. K.; Wilson, P. J.; Patch, A. M.; Wu, J.; Chang, D. K.; Cowley, M. J.; Gardiner, B. B.; Song, S.; Harliwong, I.; Idrisoglu, S.; Nourse, C.; Nourbakhsh, E.; Manning, S.; Wani, S.; Gongora, M.; Pajic, M.; Scarlett, C. J.; Gill, A. J.; Pinho, A. V.; Rooman, I.; Anderson, M.; Holmes, O.; Leonard, C.; Taylor, D.; Wood, S.; Xu, Q.; Nones, K.; Fink, J. L.; Christ, A.; Bruxner, T.; Cloonan, N.; Kolle, G.; Newell, F.; Pinese, M.; Mead, R. S.; Humphris, J. L.; Kaplan, W.; Jones, M. D.; Colvin, E. K.; Nagrial, A. M.; Humphrey, E. S.; Chou, A.; Chin, V. T.; Chantrill, L. A.; Mawson, A.; Samra, J. S.; Kench, J. G.; Lovell, J. A.; Daly, R. J.; Merrett, N. D.; Toon, C.; Epari, K.; Nguyen, N. Q.; Barbour, A.; Zeps, N.; Australian Pancreatic Cancer Genome, I.; Kakkar, N.; Zhao, F.; Wu, Y. Q.; Wang, M.; Muzny, D. M.; Fisher, W. E.; Brunicardi, F. C.; Hodges, S. E.; Reid, J. G.; Drummond, J.; Chang, K.; Han, Y.; Lewis, L. R.; Dinh, H.; Buhay, C. J.; Beck, T.; Timms, L.; Sam, M.; Begley, K.; Brown, A.; Pai, D.; Panchal, A.; Buchner, N.; De Borja, R.; Denroche, R. E.; Yung, C. K.; Serra, S.; Onetto, N.; Mukhopadhyay, D.; Tsao, M. S.; Shaw, P. A.; Petersen, G. M.; Gallinger, S.; Hruban, R. H.; Maitra, A.; lacobuzio-Donahue, C. A.; Schulick, R. D.; Wolfgang, C. L.; Morgan, R. A.; Lawlor, R. T.; Capelli, P.; Corbo, V.; Scardoni, M.; Tortora, G.; Tempero, M. A.; Mann, K. M.; Jenkins, N. A.; PerezMancera, P. A.; Adams, D. J.; Largaespada, D. A.; Wessels, L. F.; Rust, A. G.; Stein, L. D.; Tuveson, D. A.; Copeland, N. G.; Musgrove, E. A.; Scarpa, A.; Eshleman, J. R.; Hudson, T. J.; Sutherland, R. L.; Wheeler, D. A.; Pearson, J. V.; McPherson, J. D.; Gibbs, R. A.; Grimmond, S. M., Pancreatic cancer genomes reveal aberrations in axon guidance pathway genes. Nature2012, 491 (7424), 399-405.

17. Franzolin, G.; Tamagnone, L., Semaphorin Signaling in Cancer-Associated Inflammation. Int J Mol Sci2019, 20 (2). 
18. Luchino, J.; Hocine, M.; Amoureux, M.-C.; Gibert, B.; Bernet, A.; Royet, A.; Treilleux, I.; Lécine, P.; Borg, J.P.; Mehlen, P., Semaphorin 3E suppresses tumor cell death triggered by the plexin D1 dependence receptor in metastatic breast cancers. Cancer Cel/2013, 24 (5), 673-685.

19. Zhou, X.; Wahane, S.; Friedl, M. S.; Kluge, M.; Friedel, C. C.; Avrampou, K.; Zachariou, V.; Guo, L.; Zhang, B.; He, X.; Friedel, R. H.; Zou, H., Microglia and macrophages promote corralling, wound compaction and recovery after spinal cord injury via Plexin-B2. Nat Neurosci2020, 23 (3), 337-350.

20. Alto, L. T.; Terman, J. R., Semaphorins and their Signaling Mechanisms. Methods Mol Bio/2017, 1493, $1-25$.

21. Artigiani, S.; Conrotto, P.; Fazzari, P.; Gilestro, G. F.; Barberis, D.; Giordano, S.; Comoglio, P. M.; Tamagnone, L., Plexin-B3 is a functional receptor for semaphorin 5A. EMBO Rep2004, 5(7), $710-4$.

22. Pan, G. Q.; Ren, H. Z.; Zhang, S. F.; Wang, X. M.; Wen, J. F., Expression of semaphorin 5A and its receptor plexin $\mathrm{B} 3$ contributes to invasion and metastasis of gastric carcinoma. World $J$ Gastroentero/2009, 15 (22), 2800-4.

23. Malherbe, K., Tumor microenvironment and the role of artificial intelligence in breast cancer detection and prognosis. Am J Patho/2021.

24. Lin, H. J.; Lin, J., Seed-in-Soil: Pancreatic Cancer Influenced by Tumor Microenvironment. Cancers (Basel)2017, $9(7)$.

25. Quail, D. F.; Joyce, J. A., Microenvironmental regulation of tumor progression and metastasis. Nat Med2013, 19 (11), 1423-37.

26. Jiang, S. H.; Li, J.; Dong, F. Y.; Yang, J. Y.; Liu, D. J.; Yang, X. M.; Wang, Y. H.; Yang, M. W.; Fu, X. L.; Zhang, X. X.; Li, Q.; Pang, X. F.; Huo, Y. M.; Li, J.; Zhang, J. F.; Lee, H. Y.; Lee, S. J.; Qin, W. X.; Gu, J. R.; Sun, Y. W.; Zhang, Z. G., Increased Serotonin Signaling Contributes to the Warburg Effect in Pancreatic Tumor Cells Under Metabolic Stress and Promotes Growth of Pancreatic Tumors in Mice. Gastroenterology2017, $153(1), 277-291$ e19.

27. Liberti, M. V.; Dai, Z.; Wardell, S. E.; Baccile, J. A.; Liu, X.; Gao, X.; Baldi, R.; Mehrmohamadi, M.; Johnson, M. O.; Madhukar, N. S.; Shestov, A. A.; Chio, I. I. C.; Elemento, O.; Rathmell, J. C.; Schroeder, F. C.; McDonnell, D. P.; Locasale, J. W., A Predictive Model for Selective Targeting of the Warburg Effect through GAPDH Inhibition with a Natural Product. Cell Metab2017, 26 (4), 648-659 e8.

28. Donohoe, D. R.; Collins, L. B.; Wali, A.; Bigler, R.; Sun, W.; Bultman, S. J., The Warburg effect dictates the mechanism of butyrate-mediated histone acetylation and cell proliferation. Mol Cel/2012, 48 (4), 612-26.

29. Crooks, D. R.; Linehan, W. M., The Warburg effect in hominis: isotope-resolved metabolism in ccRCC. Nat Rev Uro/2018, 15 (12), 731-732.

30. Warburg, O., On the origin of cancer cells. Science1956, 123 (3191), 309-14.

31. Warburg, O., On respiratory impairment in cancer cells. Science1956, 124 (3215), 269-70.

\section{Figures}


A
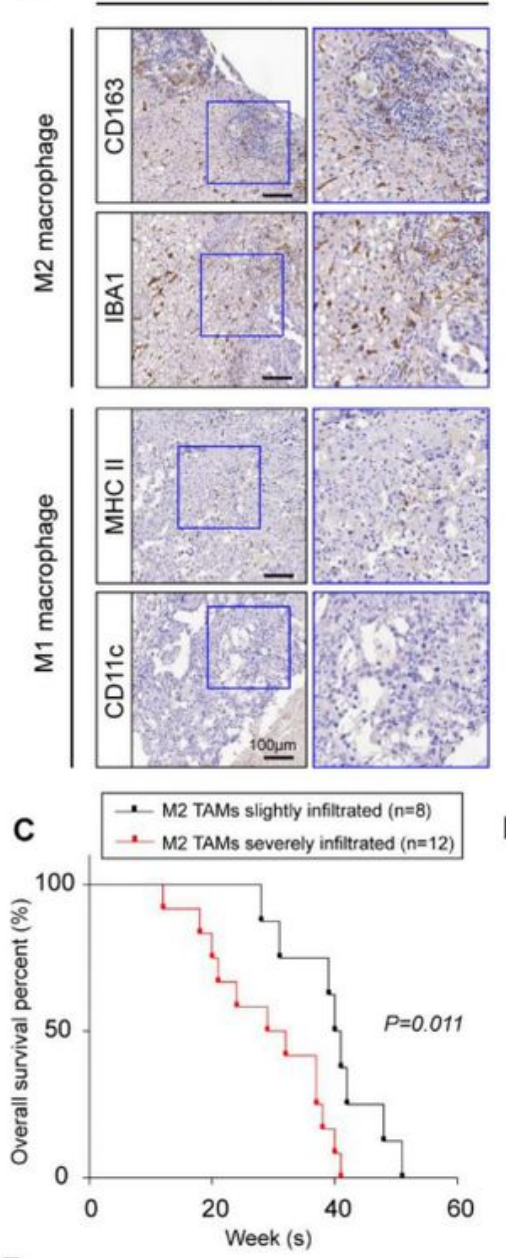

E

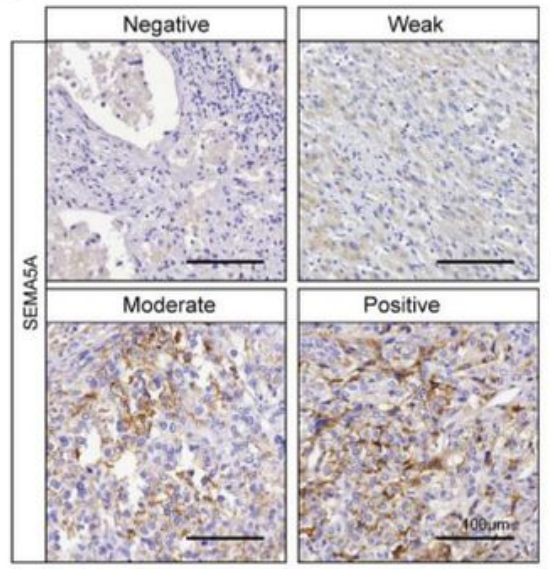

D
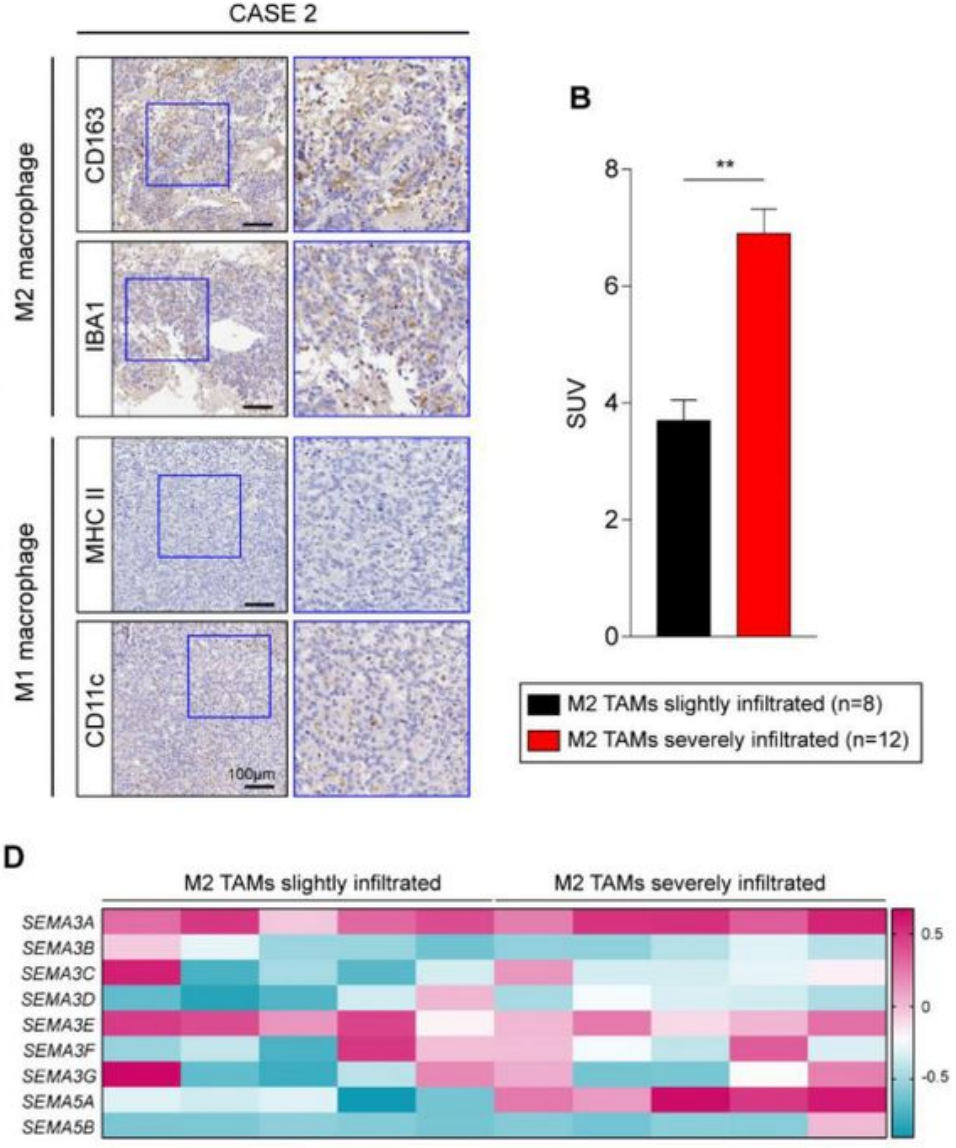

$\mathbf{F}$

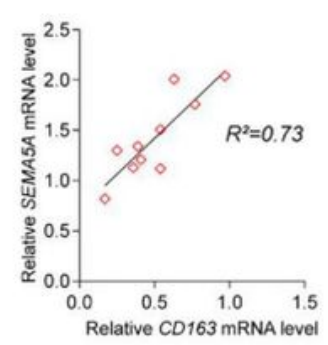

H

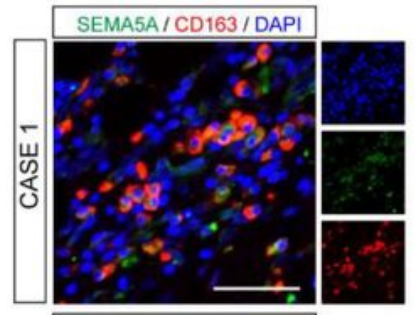

SEMA5A/CD163/DAPI

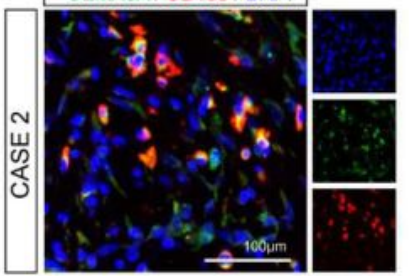

Figure 1

SEMA5A is secreted from M2 macrophages in PDAC liver metastatic niches

(A) Abundance and distribution of infiltrated TAMs in liver metastasis of PDAC were measured by IHC-P staining on M1 macrophage markers (CD163 and IBA1) or M2 macrophage markers (MHCII and CD11C) (

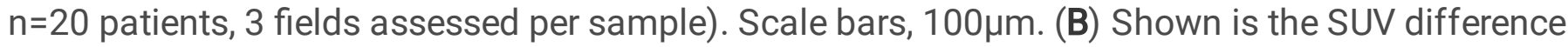


between patients bearing TAMs slightly infiltrated metastatic niches $(n=8)$ and TAMs severely infiltrated ones $(n=12)$. mean \pm s.e.m.; two tailed unpaired t-test, $* *, P<0.01$. (C) Kaplan-Meier analysis on patients bearing TAMs slightly infiltrated metastatic niches $(n=8)$ and TAMs severely infiltrated ones $(n=12)$ was performed. (D) Shown are real-time PCR measured expressions of secreted semaphorin family in TAMs slightly or severely infiltrated metastatic niches ( $n=5$ samples per group). (E) IHC-P staining on SEMA5A demonstrating its distribution in liver metastatic niches of PDAC $(n=20$ patients, 3 fields assessed per sample). Scale bars, $100 \mu \mathrm{m}$. (F-G) The relationship of CD163 expression and SEMA5A (F) or SEMA5B (G) were measured ( $n=10$ samples). (H) IF staining on SEMA5A and CD163 in PDAC liver metastatic niches displaying their co-location situations ( $\mathrm{n}=20$ patients, 3 fields assessed per sample). SEMA5A, green;

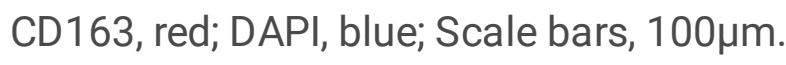


A

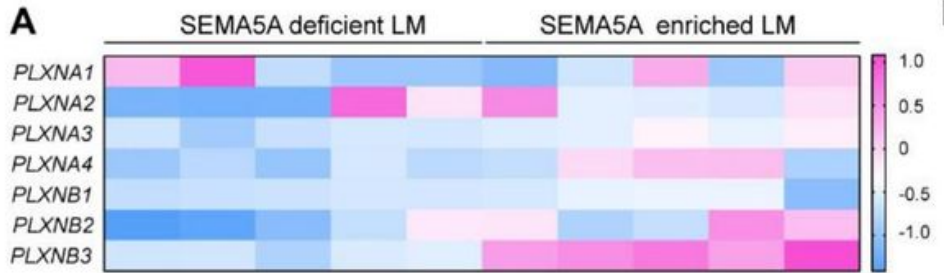

C PLXNB3 / SEMA5A/DAPI
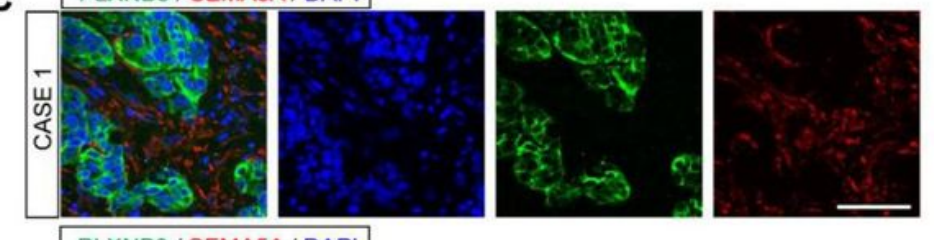

PLXNB3 / SEMA5A / DAPI
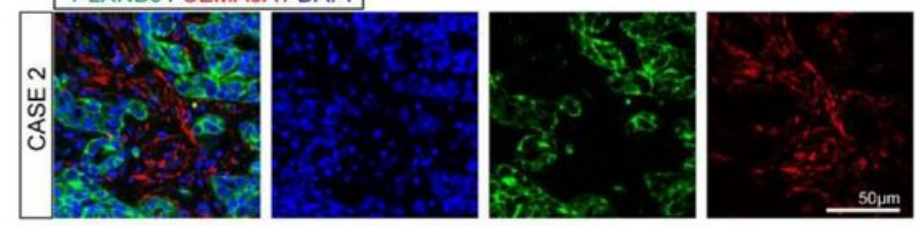

D
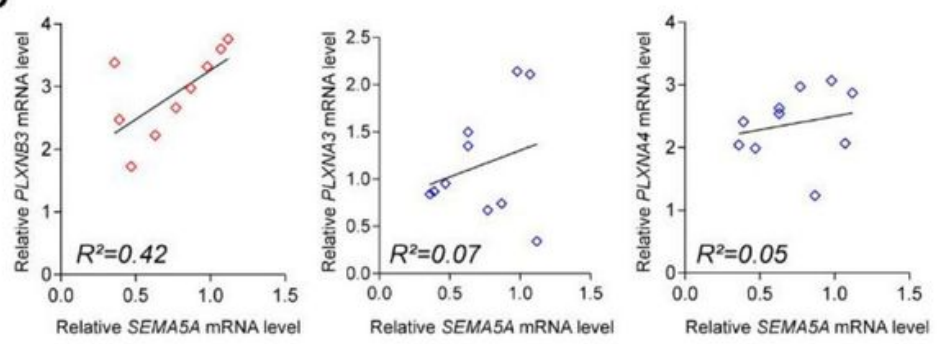

B

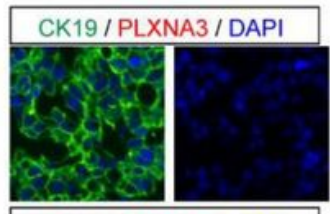

CK19/ PLXNA4 / DAPI

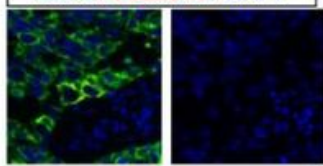

CK19/PLXNB3 / DAPI
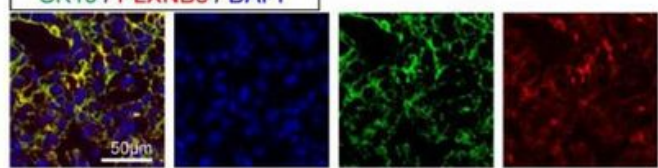

E
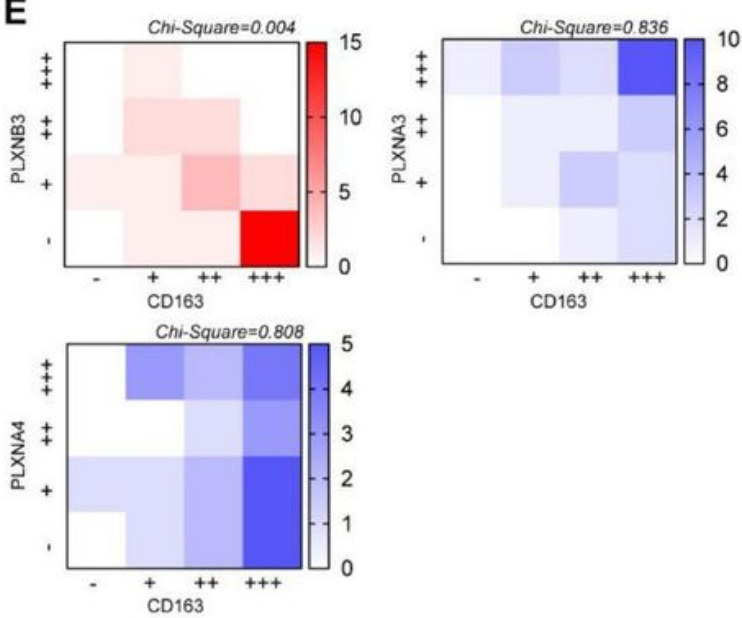

$\mathbf{F}$
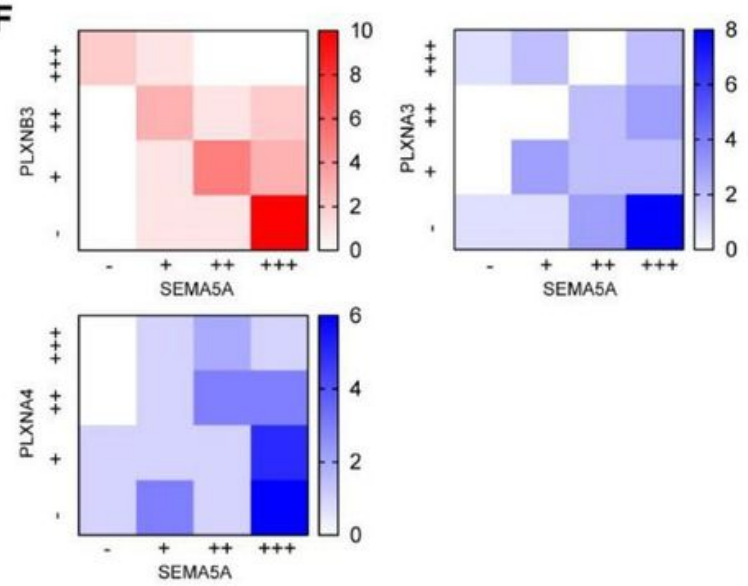

G

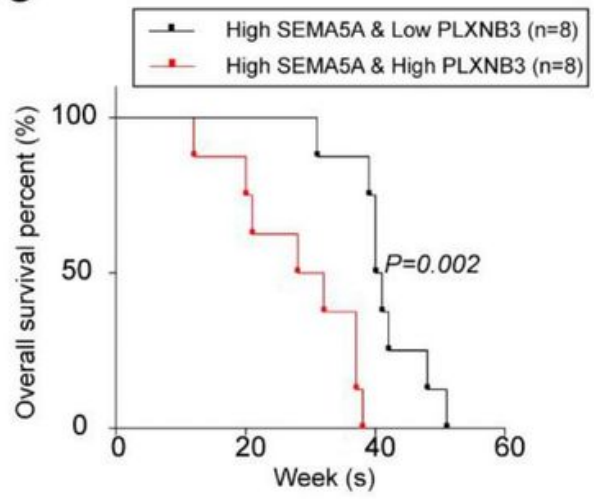

Figure 2

\section{Aberrant dual high SEMA5A and PLXNB3 expressions lead to poor prognosis}

(A) Shown are real-time PCR measured expressions of PLXN family in TAMs slightly or severely infiltrated metastatic niches. ( $n=5$ samples per group). (B) IF staining on CK19 and PLXNA3 (top), PLXNA4 (middle) or PLXNB3 (bottom) in liver metastatic niches demonstrating PLXNs locations ( $n=20$ patients, 3 fields

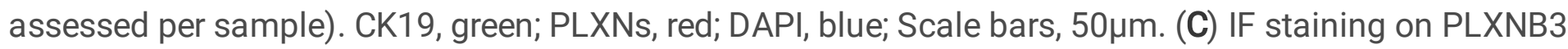


and SEMA5A in liver metastatic niches displaying their colocation relationship ( $\mathrm{n}=20$ patients, 3 fields

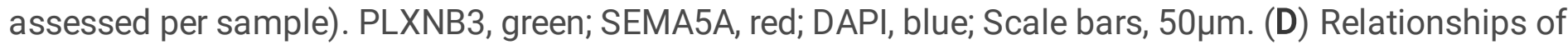
PLXNB3 (left), PLXNA3 (middle) or PLXNA4 (right) mRNA expressions and SEMA5A were measured ( $n=10$ samples). (E-F) Heatmap showing the co-expression situations of PLXNB3, PLXNA3 or PLXNA4 and CD163 (E) or SEMA5A (F) in liver metastatic niches $(n=30)$. (G) Kaplan-Meier analysis on PDAC patients bearing dual high expressions of SEMA5A and PLXNB3 or single high expression of SEMA5A in liver metastatic niches ( $\mathrm{n}=8$ patients per group).

A

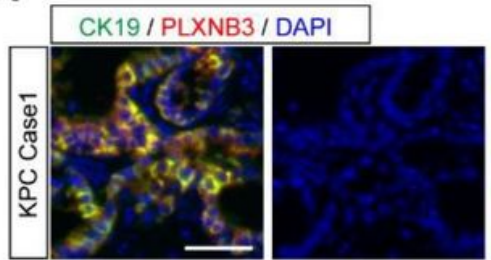

CK19 / PLXNB3 / DAPI

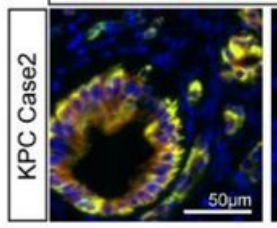

C
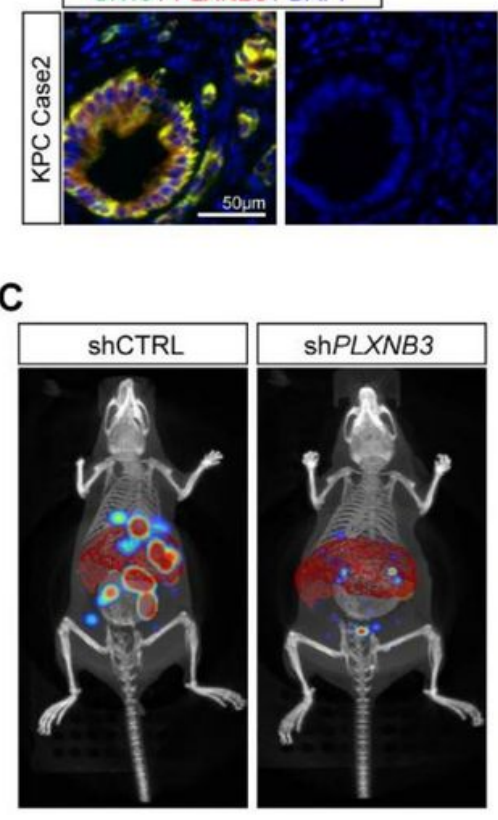
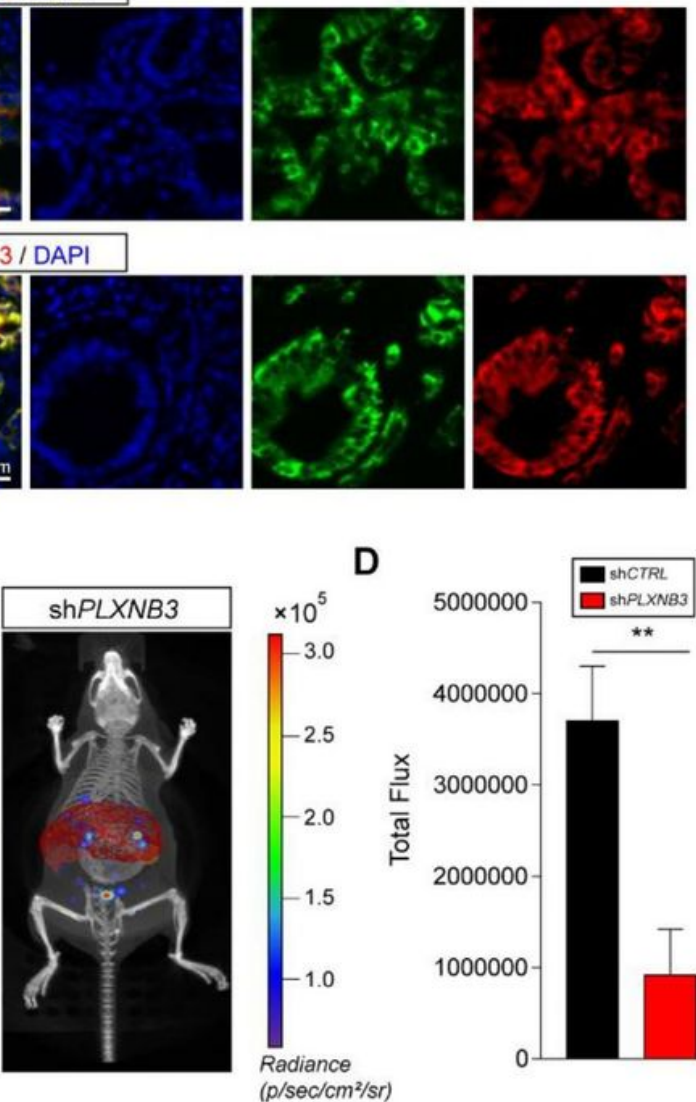

B

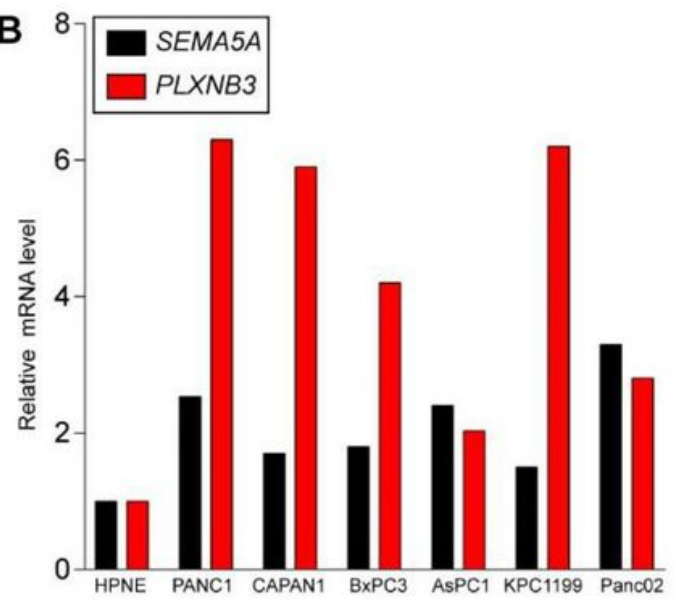

E

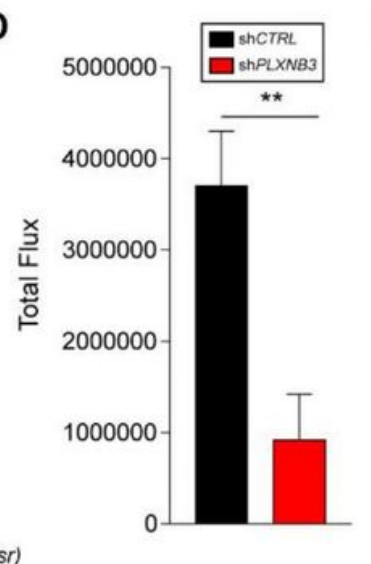

H

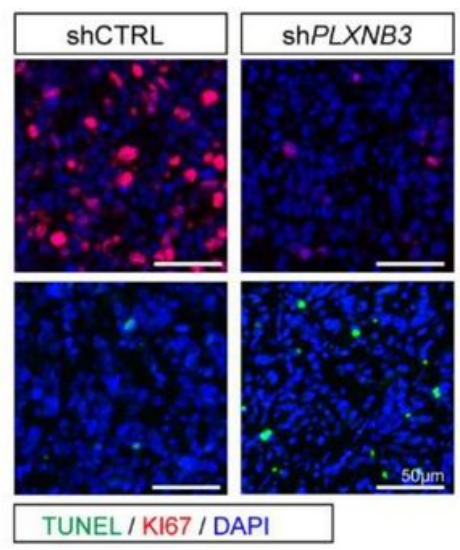

$\mathbf{F}$

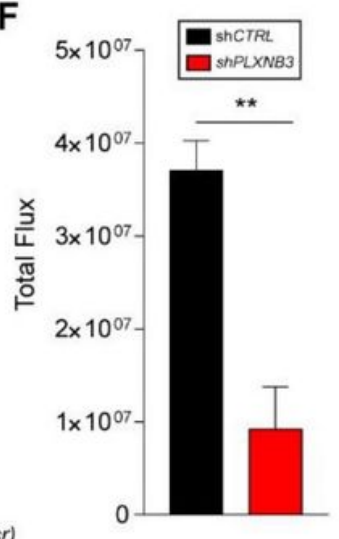

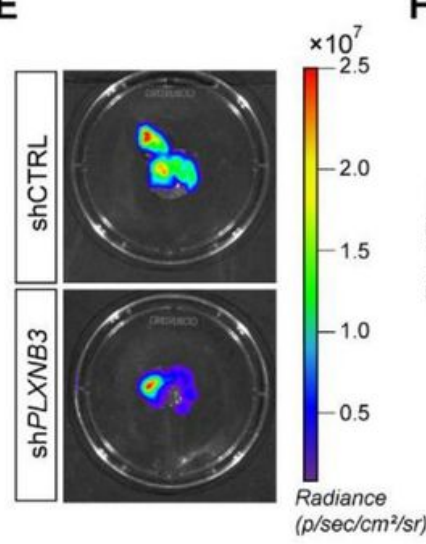

TUNEL / KI67 / DAPI
I

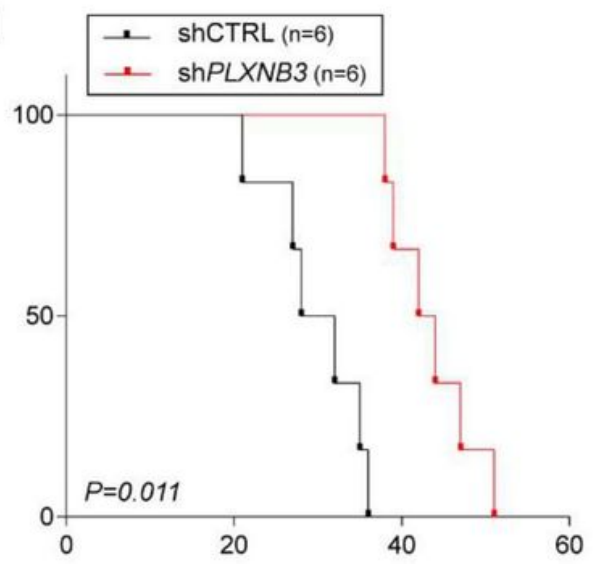

G

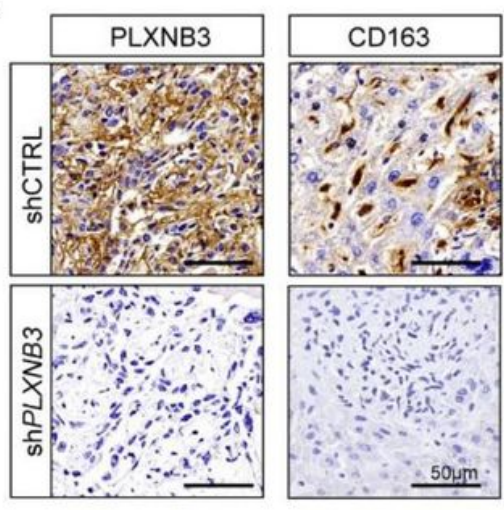

Figure 3 


\section{SEMA5A-PLXNB3 axis promotes liver metastasis in vivo}

(A) The co-expressions of CK19 and PLXNB3 were performed by IF staining in spontaneous PDAC liver metastasis KPC mouse model ( $\mathrm{n}=6$ mice, 3 fields assessed per sample). CK19, green; PLXNB3, red; DAPI,

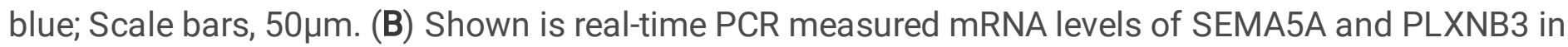
human normal pancreas epithelial cell line HPNE, human PDAC cell line PANC1, CAPAN1, BxPC3 and AsPC1 and murine PDAC cell line KPC1199 and Panc02. (C-D) CT combined in vivo imaging with organ reconstruction assessed liver metastasis burdens of intrasplenic injection mouse model $(n=6$ mice per group). Scale color bars: $5.00 \times 10^{4}-3.00 \times 10^{5}$. Reconstructed liver, red; reconstructed spleen, orange; signals of tumor niches, rainbow. mean \pm s.e.m.; two tailed unpaired t-test, $\star \star, ~ P<0.01$. (E-F) Separated KPC1199shCTRL or KPC1199shPLXNB3 invaded mouse livers were assessed via in vivo imaging system. ( $n=6$ mice per group). Scale color bars: $1.50 \times 10^{6}-2.50 \times 10^{7}$. rainbow, signals of tumor niches. mean \pm s.e.m.; two tailed unpaired t-test, $* \star, P<0.01$. (G) IHC-P staining of PLXNB3 (left) or CD163 in liver

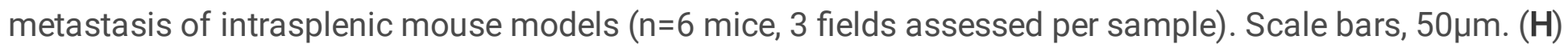
Ki67 and TUNEL staining on liver metastatic niches of intrasplenic injection mouse model were performed ( $n=6$ mice, 3 fields assessed per sample). TUNEL signals, green; Ki67, red; DAPI, blue; Scale

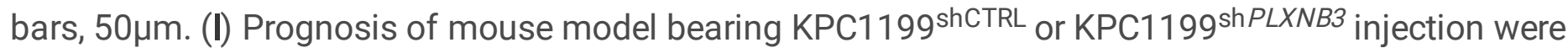
analyzed via Kaplan-Meier curve ( $n=6$ mice per group). 

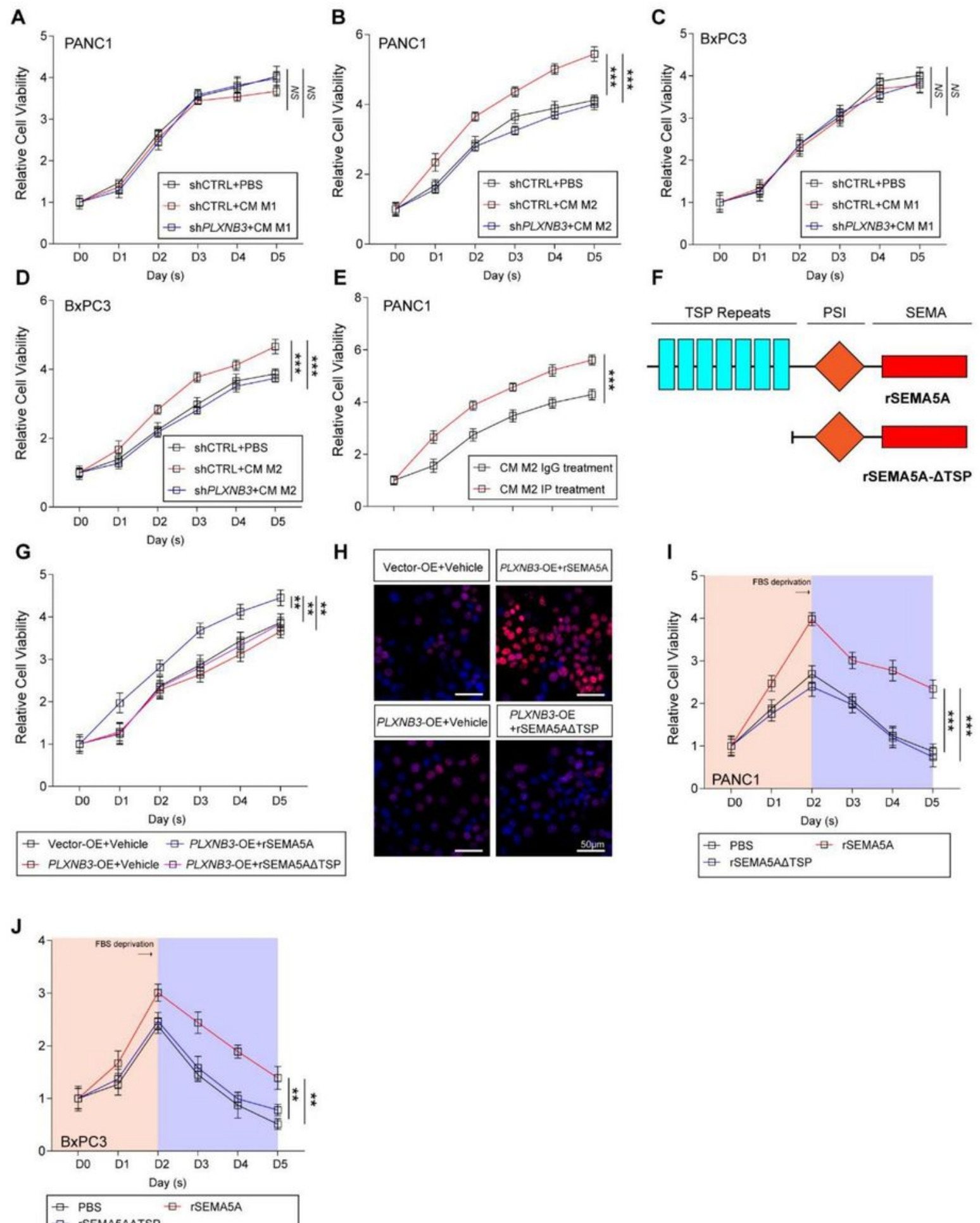

$\because$ rSEMASADTSP

\section{Figure 4}

\section{SEMA5A-PLXNB3 axis promotes liver metastasis in vivo}

(A-B) Shown is relative cell viability of PANC1 $1^{\text {shCTRL }}$ or PANC1 ${ }^{\text {sh } P L X N B 3}$ cells cultured with or without M1 TAMs (A) or M2 TAMs (B) conditional medium measured by CCK-8 kit $(n=5$ repeats per group, mean \pm s.e.m. Repeat measures ANOVA). ${ }^{\star \star *}, P<0.001$; NS., no significance. (C-D) Shown is relative cell 
viability of BXPC3 ${ }^{\text {shCTRL }}$ or BXPC3 ${ }^{\text {shPLXNB3 }}$ cells cultured with or without M1 TAMs (C) or M2 TAMs (D) conditional medium measured by CCK-8 kit ( $n=5$ repeats per group, mean \pm s.e.m. Repeat measures ANOVA). ${ }^{* \star}, P<0.001 ;$ NS., no significance. (E) Relative cell viability of PANC1 was cultured in M2 TAMs conditional medium experienced IP of SEMA5A. ( $n=5$ repeats per group, mean \pm s.e.m. Repeat measures ANOVA). ${ }^{* \star}, P<0.001$. (F) Shown is structure of SMEA5A full length and SEMA5A- $\triangle$ TSP. (G) Relative cell viability of $A S P C 1^{P L X N B 3-O E}$ in presence or absence of SEMA5A and SEMA5A- $\triangle T S P$. $(n=5$ repeats per group, mean \pm s.e.m. Repeat measures ANOVA). ${ }^{\star *}, P<0.01$. (H) EdU staining were performed on AsPC1 ${ }^{P L X N B 3-O E}$ in presence or absence of SEMA5A and SEMA5A- $\triangle$ TSP. (3 fields assessed per sample).

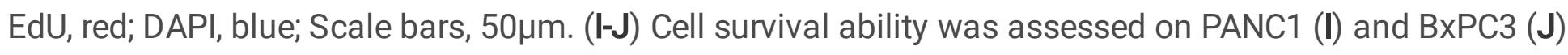
cell line with administration of rSEMA5A or SEMA5A- $\triangle T S P$. ( $n=5$ repeats per group, mean \pm s.e.m. Repeat measures ANOVA). ${ }^{\star *}, P<0.01 ; * \star \star, P<0.001$. 

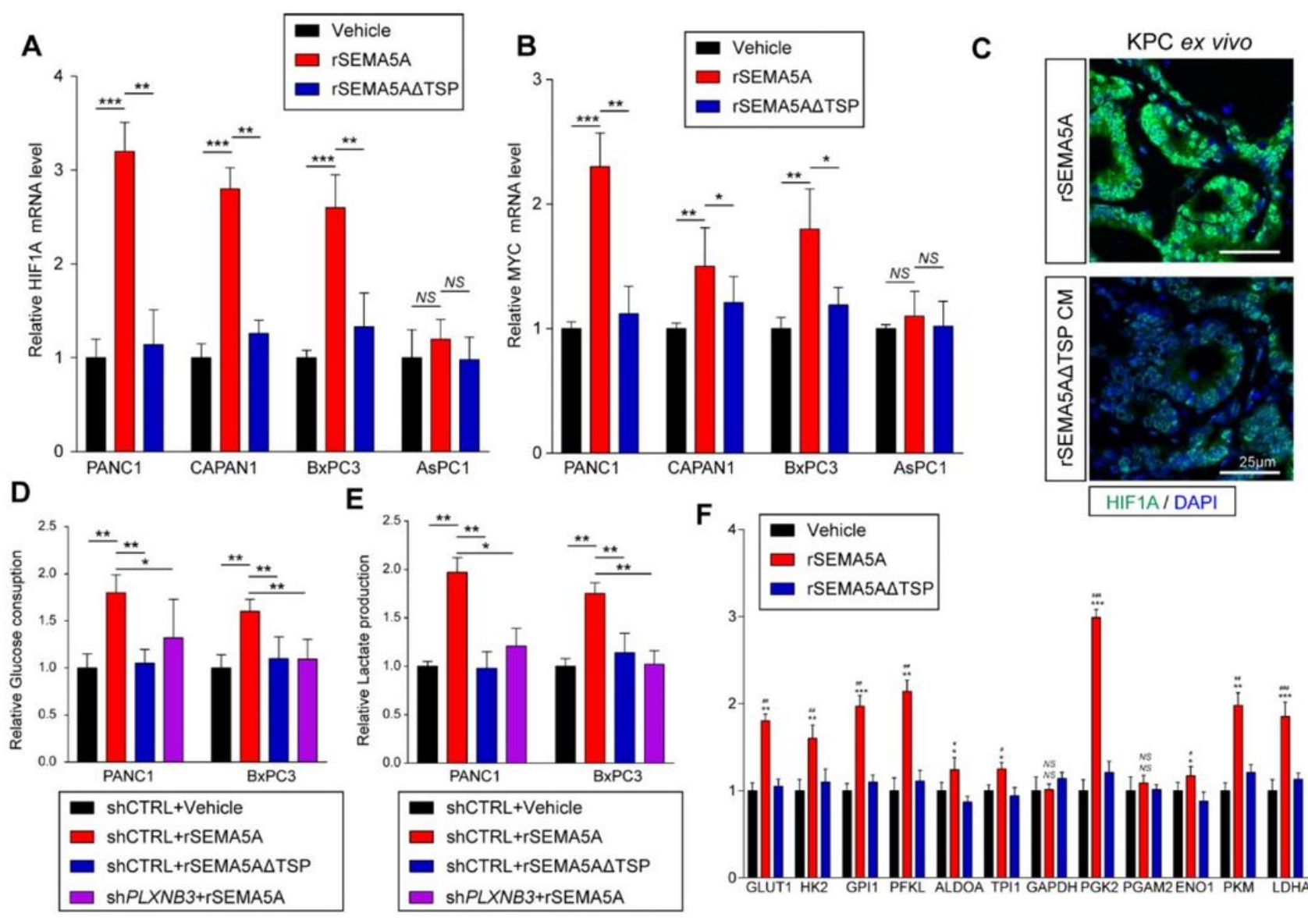

HIF1A / DAPI
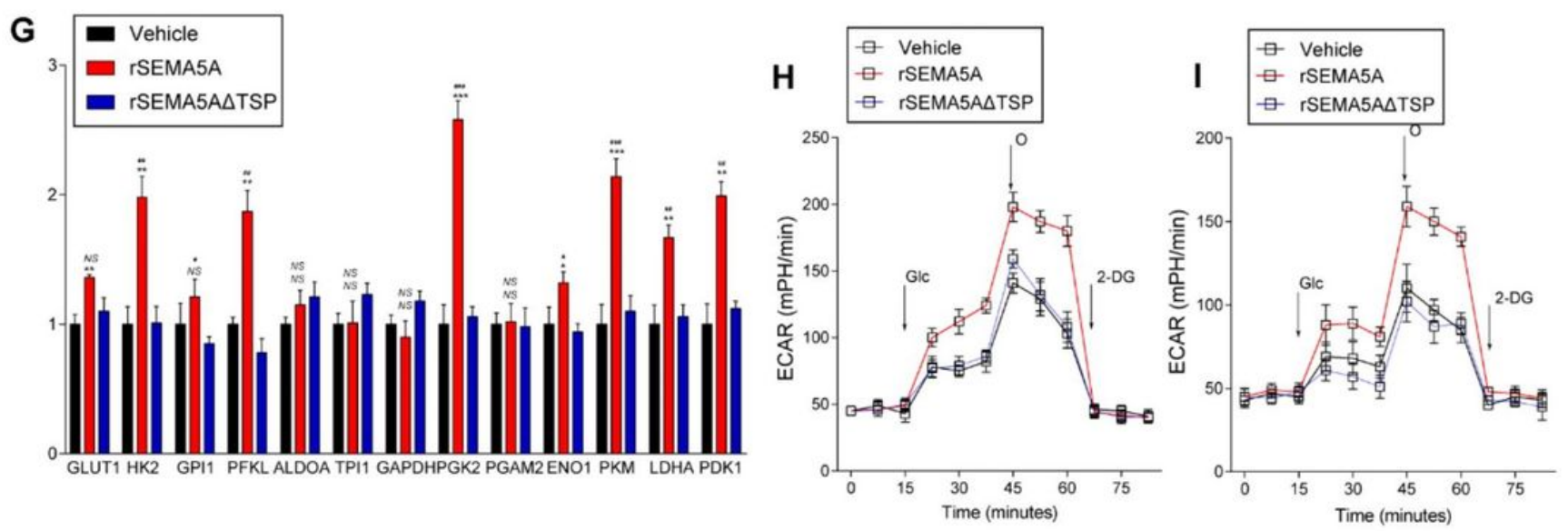

Figure 5

\section{SEMA5A-PLXNB3 axis facilitates Warburg effects via enhancing glycolysis}

(A-B) Shown is relative mRNA level of HIF1A (A) or MYC (B) affected by administration of rSEMA5A or SEMA5A- $\triangle \mathrm{TSP}$ ( $\mathrm{n}=3$ repeats per sample; mean \pm s.e.m.; two tailed unpaired t-test). $* \star, P<0.01$; $* \star \star$, $P<0.001 ;$ NS, no significant difference. (C) IF staining in KPC derived ex vivo liver metastasis tissue treated with rSEMA5A or SEMA5A- $\triangle$ TSP demonstrating the expression and distributions of HIF-1a $(\mathrm{n}=5$ mice per group, 3 fields assessed per sample). EdU, red; DAPI, blue; Scale bars, 50 $\mu$ m. (D-E) Metabolism level alterations of PANC1 and BXPC3 cell lines affected by SEMA5A-PLXN3 axis were assessed by 
relative glucose consumption (D) and lactate production $(E)$ ( $n=5$ repeats per group, mean \pm s.e.m.; two tailed unpaired t-test). ${ }^{*}, P<0.05 ; * \star, P<0.01 ; * \star *, P<0.001$. (F-G) Shown are the expression alteration of key enzymes involved in glycolysis pathway in PANC1(F) and BxPC3 (G) after stimulation by rSEMA5A or SEMA5A- $\triangle \mathrm{TSP}$ exposure ( $\mathrm{n}=3$ repeats per group, mean \pm s.e.m.; two tailed unpaired $\mathrm{t}$-test). ${ }^{*}, P<0.05$; **, $P<0.01$; ***, $P<0.001$ for rSEMA5A group; $\#, P<0.05$; \#\#, $P<0.01$; ***, $P<0.001$ for SEMA5A- $\triangle$ TSP group; NS., no significance. $(\mathbf{H}-\mathrm{I})$ Shown is relative ECARs in PANC1 $(\mathbf{H})$ or BXPC3 (I) treated with rSEMA5A or SEMA5A- $\triangle T S P$ measured by seahorse system ( $n=3$ repeats, mean \pm s.e.m.). 